\title{
Calidad morfológica y fisiológica de pepinos cultivados en diferentes concentraciones nutrimentales
}

\section{Morphological and physiological quality of cucumbers grown in different nutritional concentrations}

FERNANDO VICENTE BARRAZA-ÁLVAREZ1, 2

Cultivo de pepino en invernadero, híbrido Saber.

Foto: F.V. Barraza-Álvarez

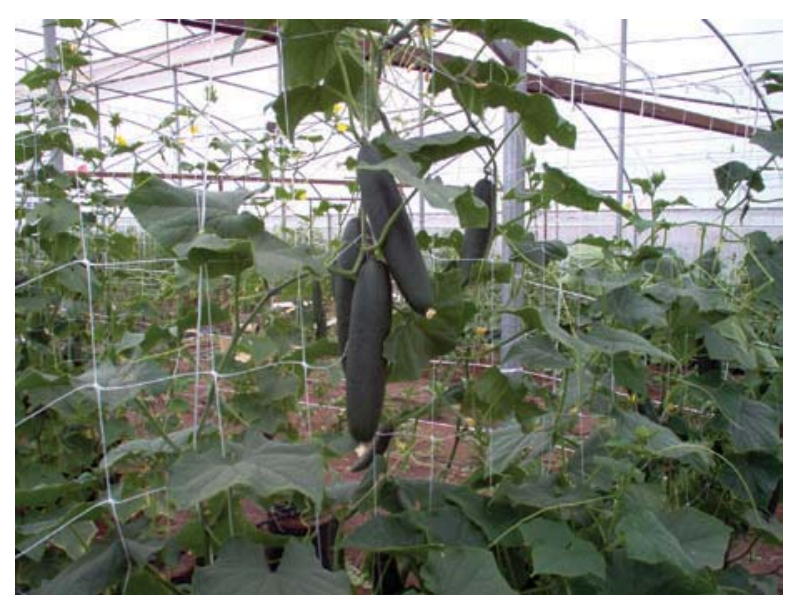

\section{RESUMEN}

Las características de calidad de los frutos de pepino están relacionadas con la nutrición mineral. De su mejor conocimiento depende el manejo poscosecha efectuado para mantener la calidad final. Por lo anterior, se hizo una investigación en la Universidad Autónoma Chapingo (México), con el objetivo de determinar cuál de las concentraciones de la solución nutritiva universal de Steiner más utilizadas por los agricultores, tiene mejor efecto sobre las principales características de calidad de los frutos de pepino cultivado en invernadero. Se evaluaron las variables: longitud, diámetro ecuatorial, firmeza, grados Brix, acidez total titulable (ATT), clorofila total, luminosidad y color. Se utilizó diseño de bloques al azar con cuatro tratamientos: $25 \%, 75 \%, 125 \%$ y $175 \%$ de la solución nutritiva universal de Steiner. De acuerdo con los resultados obtenidos, la longitud y diámetro ecuatorial de frutos tuvieron mayores valores para 175\% de concentración, con diferencias estadísticas significativas respecto a las demás concentraciones. Este tratamiento superó también a los demás en firmeza, contenido total de clorofila, luminosidad y color. Con la concentración del $25 \%$ no se presentó la primera cosecha de frutos (49 días después del trasplante), y hubo valores muy bajos de ATT (1,68\%). Respecto a grados Brix no hubo diferencias entre tratamientos.

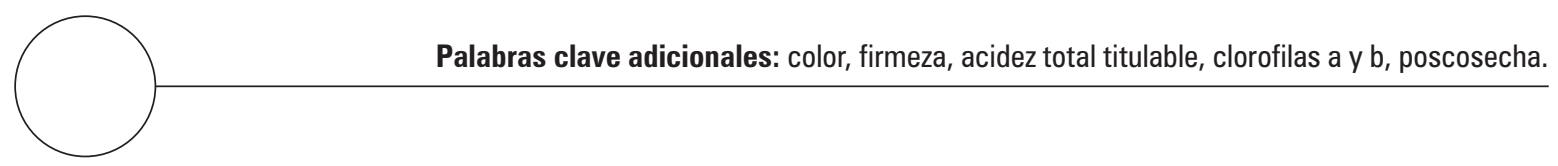

Facultad de Ciencias Agrícolas, Programa de Ingeniería Agronómica, Universidad de Córdoba, Montería (Colombia).

2 Autor para correspondencia.fbarraza@correo.unicordoba.edu.co 


\section{ABSTRACT}

The quality characteristics of cucumber fruits are related to mineral nutrition. Postharvest handling affects the final quality. Therefore, research was conducted by the Universidad Autónoma Chapingo (México) in order to determine which of the concentrations of Steiner's universal nutrient solution, which is most used by farmers, has the best effect on the main characteristics of quality in cucumber fruits cultivated in a greenhouse. The following variables were evaluated: length, equatorial diameter, firmness, Brix degrees, total titratable acidity (TTA), whole chlorophyll content, brightness and color. A randomized block design was used with four treatments: $25 \%, 75 \%, 125 \%$ and $175 \%$ of Steiner's universal nutrient solution. According to the obtained results, the length and equatorial diameter of the fruits reached higher values with a $175 \%$ concentration, with significant statistical differences with respect to the other concentrations. This treatment also exceeded the others in firmness, whole chlorophyll content, brightness and color. With the $25 \%$ concentration, the first fruit harvest was not obtained (49 days after the transplant) and there were very low TTA values $(1,68 \%)$. There were no differences between the treatments for the Brix degrees.

Additional key words: Color, firmness, titratable acidity, a and b chlorophylls, postharvest.

Fecha de recepción: 04-02-2015

Aprobado para publicación: 28-05-2015
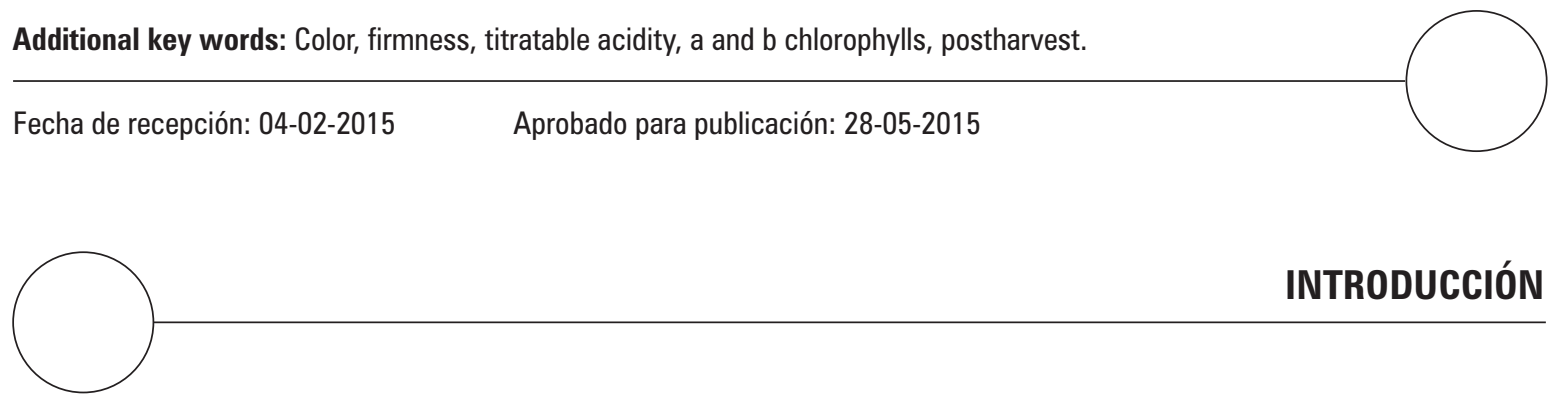

El pepino (Cucumis sativus L.) es una de las hortalizas cucurbitáceas más conocidas. Se cultiva en casi todo el mundo principalmente para consumo de sus frutos no climatéricos en estado inmaduro. En $100 \mathrm{~g}$ de parte comestible, los pepinos poseen alto contenido de agua $(96,7 \%)$ y pocas calorías (9); además contienen vitamina A (20 UI), vitamina B1 (0,02 $\mathrm{mg})$, vitamina B2 (0,02 mg), vitamina B3 (0,1 mg), vitamina C (8 $\mathrm{mg}$ ), y minerales como calcio (7 $\mathrm{mg}$ ), potasio (147 mg), hierro (0,3 mg), fósforo (30 mg) y magnesio (13 mg) (Caicedo, 1993; Tsuchida et al., 2010; Kazemi, 2013).

El consumo del pepino lo ubica como la cuarta hortaliza más importante del mundo, después del jitomate (Solanum lycopersicum L.), repollo (Brassica oleracea L. var. capitata) y cebolla (Allium cepa L.). Se utiliza tanto en estado fresco como industrial (pepinillos o "pickles"). También tiene amplio uso en cosmetología y salud, en la fabricación de jabones, cremas y productos que aprovechan sus propiedades como emoliente, diurético, depurativo, laxante y calmante, así como sus efectos en tratamientos de aclaramiento de la piel y manchas, reducción de ojeras y nutrición del cuero cabelludo (Qureshi et al., 2010; Abu et al., 2013).

Junto con la sandía (Citrullus vulgaris Shard.), melón (Cucumis melo L.) y jitomate (S. lycopersicum), son las especies de hortalizas de frutos más importantes que exporta México al mercado internacional. En el año 2013 el país ocupó el primer lugar en exportaciones de pepinos, con 368.383 t, cuyo valor fue de \$US 185 millones (Banco de México, 2013).

La buena calidad de los frutos de pepino está principalmente representa en la uniformidad de la forma, firmeza, color verde oscuro del exocarpo, tamaño y ausencia de defectos de crecimien- 
to o manejo, así como de pudriciones y amarillamientos (Suslov y Cantwell, 2012), que son características que dependen de las condiciones de manejo dadas al cultivo, y que una vez cosechados los frutos en madurez comercial, comienzan a experimentar cambios a nivel morfológico y fisiológico, especialmente en el metabolismo, lo que influye en la apariencia y calidad integral del producto que llega al consumidor final (Gruda, 2005; Moreno et al., 2013). Por ello, en vista de que el pepino es un cultivo de rápido crecimiento y sus frutos son los principales centros de demanda e influyen en gran medida en la acumulación y partición de materia seca (Gómez et al., 2006; Barraza, 2012), el manejo integrado de la nutrición mineral para aumentar el rendimiento y obtener un producto de buena calidad se ha reportado como una necesidad prioritaria de investigación en los países desarrollados, con el fin de estandarizar criterios y mejorar el manejo poscosecha, especialmente en los sistemas modernos de horticultura protegida y que además hay variación en el comportamiento de las características de calidad de los frutos y la velocidad de su deterioro de acuerdo con los sistemas de cultivo empleados (Moreno et al., 2013), y que los estudios en esta temática específica son pocos y antiguos (Abu et al., 2013).

Debido a que en México la nutrición mineral del cultivo de pepino en invernadero se suministra principalmente con base en la solución nutritiva universal de Steiner, utilizada a diferentes concentraciones de acuerdo con el criterio de los productores, sin tener en cuenta a qué concentración se presenta mejor comportamiento de la calidad de los frutos, la presente investigación se hizo con el objetivo de determinar en cuál de las concentraciones más utilizadas de dicha solución se obtienen mejores características como longitud, diámetro, firmeza, grados Brix, color, acidez total titulable, contenido de clorofila total, luminosidad y color, que son parámetros de gran interés en estudios de poscosecha y de importancia para mantener e incrementar el volumen de comercialización del país.

\section{MATERIALES Y MÉTODOS}

\section{Localización}

La investigación se hizo entre abril y agosto de 2006 en invernadero del campo experimental de la Universidad Autónoma Chapingo, México, a $19^{\circ} 29^{\prime} \mathrm{N}, 98^{\circ} 53^{\prime} \mathrm{W}$ y altitud de $2.250 \mathrm{msnm}$. Dentro del invernadero hubo temperatura promedio de $21,8^{\circ} \mathrm{C}$, máxima promedio de $27,2^{\circ} \mathrm{C}$, mínima promedio de $14,5^{\circ} \mathrm{C}$ y humedad relativa promedio de $87,5 \%$.

\section{Obtención de plántulas y trasplante}

Se sembraron semillas del híbrido de pepino Saber ${ }^{\circledR}$ en bandejas de poliestireno expandido de 200 cavidades. El sustrato utilizado fue turba $\left(\right.$ Peat-Moss ${ }^{\circledR}$ ). En cada cavidad se sembró una semilla. A los 25 d después de la siembra se hizo el trasplante de plántulas con la primera hoja verdadera expandida, a bolsas de polietileno de color negro de $0,06 \mathrm{~m}^{3}$ de volumen llenadas con roca volcánica (tezontle rojo). En cada bolsa se trasplantó una plántula.

\section{Diseño experimental y conducción de la investigación}

Se utilizó diseño experimental en bloques al azar con cuatro tratamientos y tres repeticiones, en un área total de $99 \mathrm{~m}^{2}$. Las plantas se ubicaron a 1,5 m entre hileras y $0,3 \mathrm{~m}$ entre plantas. Los tratamientos ( $\mathrm{Tn}$ ) fueron cuatro concentraciones de la solución nutritiva universal de Steiner (T1: 25\%, T2: 75\%, T3: $125 \%$ y T4: $175 \%$ ), cuya composición para $100 \%$ de concentración es: $\mathrm{NO}_{3}^{-}, 12 ; \mathrm{H}_{2} \mathrm{PO}_{4}^{-}, 1 ; \mathrm{SO}_{4}^{-}, 7 ; \mathrm{Ca}^{2+}, 9 ; \mathrm{Mg}^{2+}, 4$ $\mathrm{y} \mathrm{K}^{+}, 7$ meq L $\mathrm{L}^{-1}$ (Barraza, 2012).

El suministro de soluciones nutritivas de acuerdo con los tratamientos se hizo mediante sistema hidropónico abierto con riego por goteo, así: durante 30 días después del trasplante (ddt) se aplicaron $150 \mathrm{~mL}$ diarios de solución nutritiva por planta, en tres riegos de $50 \mathrm{~mL}$; de los 31 a los 60 
ddt se aplicaron $300 \mathrm{~mL}$ diarios por planta, en tres riegos de $100 \mathrm{~mL}$; del 61 al $135 \mathrm{ddt}$, se aplicaron $600 \mathrm{~mL}$ diarios por planta distribuidos en tres riegos de $200 \mathrm{~mL}$. El intervalo entre riegos fue de $4 \mathrm{~h}$.

El cultivo se manejó sin podas con tutorado en espaldera a partir de los $15 \mathrm{~d}$ después del trasplante (ddt) con malla plástica de cuadrícula de $10 \mathrm{~cm}$.

\section{Variables estudiadas}

Se tomaron medidas de las siguientes variables durante el periodo de formación de frutos, cada 8 d en el Laboratorio de Fisiología de Poscosecha de la Universidad Autónoma Chapingo: longitud y diámetro ecuatorial del fruto (en $\mathrm{cm}$ ); firmeza (en N), con penetrómetro digital Mecmesin (Mecmesin Ltd., Slinfold, UK), grados Brix, con refractómetro digital Atago PAL-1 (Atago Co. Ltd., Bellevue, WA), acidez total titulable (\% de ácido málico), mediante titulación con $\mathrm{NaOH}$ $0,1 \mathrm{~N}$; clorofilas a y b, extraídas mediante la metodología de acetona y cuantificadas con lecturas de absorbancia (A) a 645 y $652 \mathrm{~nm}$, respectivamente, con espectrofotómetro Spectronic 20 (Thermo Fisher Scientific Inc., Madison, WI). Se calculó el contenido de clorofila total (CT) con la expresión de Harborne (1984):

$$
\mathrm{CT}\left(\mathrm{mg} \mathrm{L}^{-1}\right)=20,2 \mathrm{~A}_{645}+8,02 \mathrm{~A}_{663}
$$

La luminosidad $\left(\mathrm{L}^{*}\right)$ y color (coordenadas de cromaticidad $\mathrm{a}^{*} \mathrm{y}^{*}$ ) se determinaron mediante colorímetro digital ColorTec PCM (Color Tec Associates, Inc., Clinton, NJ). Para todas las variables indicadas se obtuvo el promedio de medidas efectuadas a tres frutos de tres plantas seleccionadas al azar en las tres hileras centrales de cada repetición.

\section{Procesamiento estadístico}

Para las variables estudiadas se hizo análisis de varianza y prueba de comparación de medias de Tukey $(P \leq 0,05)$ con software SAS 9.0 (Cary,
N.C.). Para modelar el comportamiento de las variables longitud y diámetro ecuatorial del fruto, se aplicó el procedimiento de regresión no lineal (NLIN) mediante el software SAS 9.0, y se seleccionó el modelo logístico, de acuerdo con Marcelis (1992):

$$
y=\frac{A}{\left(1+B \cdot e^{-C \cdot x}\right)}
$$

Donde

$y=$ variable respuesta (longitud y diámetro ecuatorial, en función de $x$ ),

$x=$ tiempo $(d d t)$,

$A=$ asíntota, valor de $y$ cuando $x$ tiende a infinito,

$B \cdot e^{-c x}$ : factor de amortiguamiento, donde $B$ corresponde a un factor de amplitud, no tiene significado biológico y solo toma lugar en el tiempo inicial, cuando $x=0 ; C=$ factor de estabilidad relacionado con el valor de $x$ y $e=$ número Euler.

\section{RESULTADOS Y DISCUSIÓN}

\section{Longitud y diámetro ecuatorial del fruto}

Se encontró una representación gráfica sigmoide (figuras 1 y 2), reportada para la mayoría de cultivos y órganos vegetales aislados (con muy pocas excepciones en la naturaleza, por ejemplo Actinidia deliciosa) que de acuerdo con Ando et al. (2012) se manifiesta como aumento de peso, volumen, área o longitud por unidad de tiempo, desde el momento de la antesis hasta que los frutos alcanzan la madurez. Similares resultados obtuvieron para los cultivares de pepino 'Brillante' (Davies y Kempton, 1976), 'Farbio' (Schapendonk y Challa, 1980), 'Corona' (Marcelis, 1992), 'Vlaspik' (Ando y Grumet, 2010), 'Young' Vlaspik (Ando et al., 2012), 'Hassan local' (Anjanappa et al., 2012), 'Chinese long' (Yong et al., 
2013) y 'Mostar' (Kazemi, 2013), lo que indica que corresponde a un comportamiento propio de la especie (Schapendonk y Brouwer, 1984).

Ambas variables se ajustaron al modelo logístico, proporciones de variabilidad cercanas a la unidad, lo que confiere certidumbre para estimar el proceso de crecimiento ocurrido en el fruto a través del ciclo del cultivo (tabla 1). Igual comportamiento se obtuvo para otras variables del crecimiento de pepino como acumulación de materia seca de tallos, materia seca de hojas, materia seca de zarcillos y materia seca total (Barraza, 2012).

Inicialmente, entre los 36 y $41 \mathrm{ddt}$ (figuras 1 y 2), el crecimiento del fruto en longitud y diámetro ecuatorial tuvo valores bajos, posiblemente por el aumento de la respiración que acompaña la fase inicial de división celular que ocurre cuando el ovario es fecundado. Posteriormente, entre los 42 y 50 ddt se observó una fase de rápido crecimiento que, de acuerdo con Ando et al. (2012), ocurre de manera exponencial, con expansión celular que se manifiesta en rápido incremento del tamaño del fruto.

Respecto al inicio de cosecha, de acuerdo con Ando y Grumet (2010) y Ando et al. (2012) ocurre a mediados o al final de la fase exponencial de crecimiento, lo que podría depender del genotipo y las condiciones ambientales. Para los tratamientos con solución al 75\%, 75\% y $125 \%$ dicho evento ocurrió a los 49 ddt (final de la fase exponencial). Para el tratamiento con solución del $25 \%$, no se presentó la primera cosecha en la fecha indicada, lo que podría estar relacionado con la menor cantidad de nutrimentos aplicada con dicha concentración (Verheul et al., 2013).

Con relación al efecto de las soluciones nutritivas sobre el crecimiento del fruto, a medida que aumentó la concentración, lo hicieron también la longitud y el diámetro ecuatorial, con diferencias estadísticas significativas (tabla 2).

Tabla 1. Modelos logísticos para estimar crecimiento en longitud y diámetro ecuatorial de frutos de pepino cultivados con cuatro concentraciones de la solución nutritiva universal de Steiner.

\begin{tabular}{|c|c|c|}
\hline Tratamiento & Variable del crecimiento & PV \\
\hline \multicolumn{3}{|c|}{ Longitud del fruto $(\mathrm{cm})$} \\
\hline $\mathrm{T} 1$ & $y=1512,1 /\left(1+51975,5 e^{-0,98510^{*} d t}\right)$ & 0,98 \\
\hline $\mathrm{T} 2$ & $y=25,2384 /\left(1+265934 e^{-0,2906^{*} d t}\right)$ & 0,97 \\
\hline T3 & $y=25,7076 /\left(1+6967810 e^{-0,3265^{*} d d t}\right)$ & 0,96 \\
\hline $\mathrm{T} 4$ & $y=26,0088 /\left(1+7775534 e^{-0,3978^{*} d d t}\right)$ & 0,99 \\
\hline \multicolumn{3}{|c|}{ Diámetro ecuatorial del fruto $(\mathrm{cm})$} \\
\hline $\mathrm{T} 1$ & $y=514,4 /\left(1+114785 e^{-0,1340^{*} d d t}\right)$ & 0,95 \\
\hline $\mathrm{T} 2$ & $y=6,7867 /\left(1+30848,4 e^{-0,2248^{*} d d t}\right)$ & 0,96 \\
\hline T3 & $y=6,029 /\left(1+848830 e^{-0,3264^{*} d d t}\right)$ & 0,99 \\
\hline T4 & $y=6,2642 /\left(1+18367764 e^{-0,4112^{*} d d t}\right)$ & 0,98 \\
\hline
\end{tabular}

y: variable del crecimiento (longitud y diámetro ecuatorial del fruto); ddt: días después del trasplante; T1: 25\%, T2: 75\%; T3: 125\%; T4: $175 \%$ de la solución nutritiva de Steiner. PV: proporción de variabilidad que indica la confiabilidad del modelo $=$ suma de cuadrados de regresión/suma de cuadrados total. 


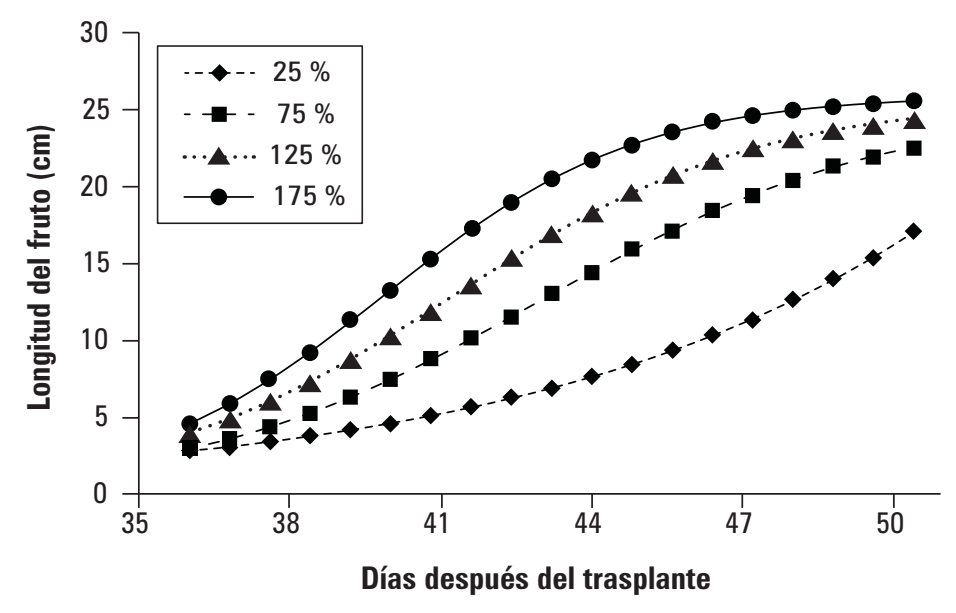

Figura 1. Longitud de frutos de pepino cultivado en cuatro concentraciones de la solución nutritiva universal de Steiner.

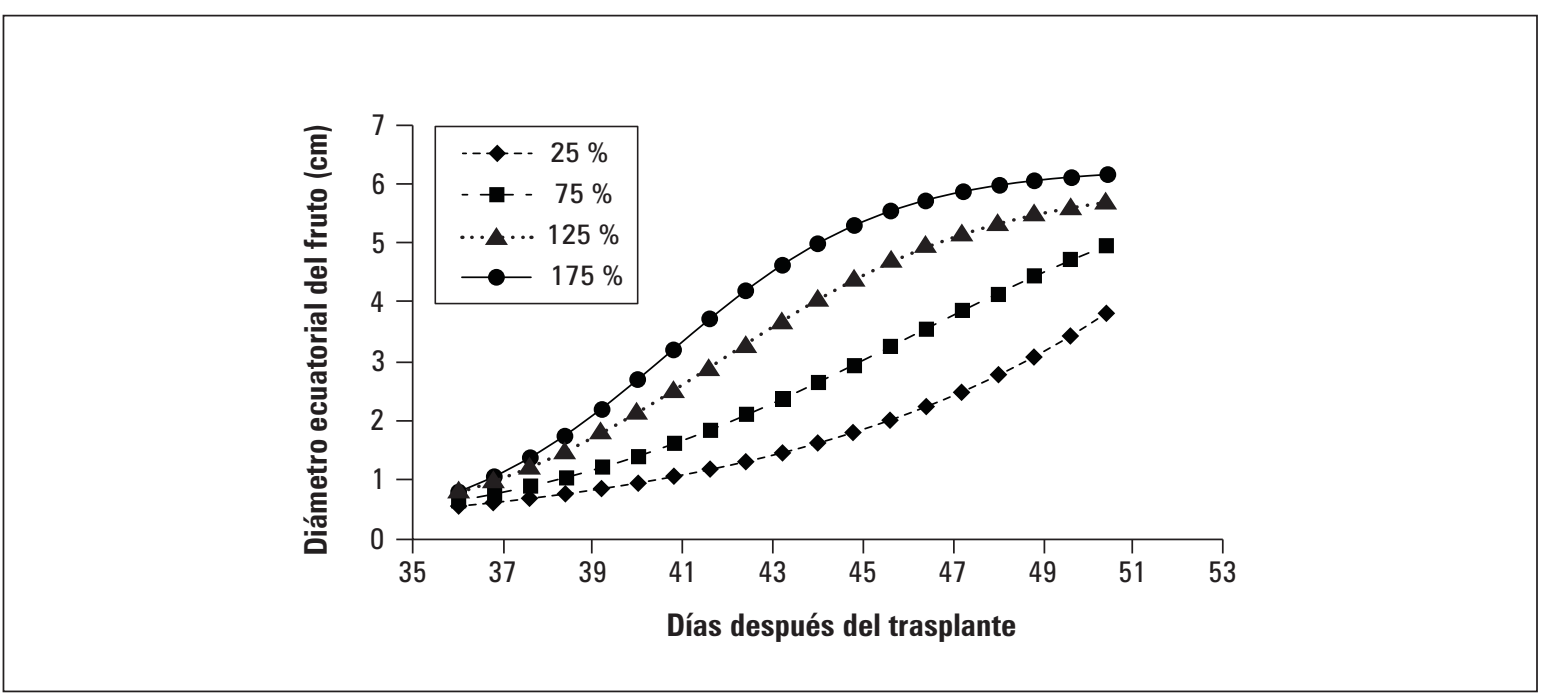

Figura 2. Diámetro ecuatorial de frutos de pepino cultivado en cuatro concentraciones de la solución nutritiva universal de Steiner.

Con el 175\% de concentración se obtuvieron mayores promedios para ambas variables (tabla 2), lo que indica que además de la división y expansión celular, así como la consecuente especialización celular en tejidos y órganos, es importante la interacción que estos procesos tienen con los factores bióticos y abióticos del ambiente, entre los que se destacan las condiciones nutrimentales que tienen lugar durante el desarrollo vegetal, las cuales modulan también el crecimiento del ova- rio y contribuyen con el aumento del rendimiento y calidad del pepino, especialmente cuando se hace un manejo integrado de la nutrición bajo condiciones de invernadero (Anjanappa et al., 2012). Al respecto indican estos autores que esto puede ser atribuido al microclima favorable que prevalece en el invernadero y al manejo integrado de la nutrición, especialmente la mayor cantidad aplicada de nitrógeno, fósforo y potasio, que contribuyen a la mejor utilización de la radiación 
Tabla 2. Comparación de medias de longitud y diámetro ecuatorial del fruto de pepino, en cuatro concentraciones de la solución nutritiva universal de Steiner.

\begin{tabular}{|c|c|c|c|c|c|}
\hline ddt & T1 & T2 & T3 & T4 & CV \\
\hline \multicolumn{6}{|c|}{ Longitud $(\mathrm{cm})$} \\
\hline 36 & $2,73 \mathrm{~b}$ & $2,93 \mathrm{~b}$ & $3,97 a b$ & 4,57 a & 13,76 \\
\hline 42 & $5,40 d$ & $10,83 \mathrm{c}$ & $14,50 \mathrm{~b}$ & $18,17 \mathrm{a}$ & 8,10 \\
\hline 49 & $15,60 \mathrm{c}$ & $21,50 \mathrm{~b}$ & $23,83 a b$ & 25,33 a & 6,25 \\
\hline \multicolumn{6}{|c|}{ Diámetro ecuatorial $(\mathrm{cm})$} \\
\hline 36 & 0,55 a & 0,65 a & 0,78 a & 0,80 a & 15,52 \\
\hline 42 & $1,13 d$ & $1,97 \mathrm{c}$ & $3,10 \mathrm{~b}$ & 3,97 a & 7,68 \\
\hline 49 & $3,57 d$ & $4,50 \mathrm{c}$ & $5,50 \mathrm{~b}$ & $6,07 \mathrm{a}$ & 3,64 \\
\hline
\end{tabular}

T (tratamientos): T1: 25\%, T2: 75\%; T3: 125\%; T4: 175\% de la solución nutritiva de Steiner; ddt: días después del trasplante; CV: coeficiente de variación; DMS: diferencia mínima significativa. Promedios con letras distintas, dentro de una misma fila, indican diferencia significativa según la prueba de Tukey $(P \leq 0,05)$.

solar, nutrimentos y agua para la síntesis de fotosintatos, hormonas (como ácido indol acético y ácido giberélico), aminoácidos y vitaminas que contribuyen también a la rápida multiplicación y elongación de células, que se manifiestan además en mejor crecimiento radicular y crecimiento vegetativo, incluyendo otras variables del crecimiento como altura de planta y número de hojas y guías primarias y secundarias.

\section{Firmeza}

Mostró incremento a través del tiempo y presentó mayores valores con el aumento de la concentración de la solución nutritiva, de manera que con el $175 \%$ la firmeza de los frutos fue superior en grado significativo con respecto a los tratamientos al $25 \%$, $75 \%$ y $125 \%$ (tabla 3 ).

Con la solución al 25\% no se encontraron frutos a los 36 ddt (tabla 3), lo que demuestra que dicha concentración suministró nutrimentos en cantidad insuficiente respecto a las demás, con lo que se afectó la entrada a producción del genotipo, bastante relacionada con un alto índice de área foliar (Den Nijs, 1980), del cual depende el suministro de fotoasimilados que se utilizan para crecimiento del fruto y metabolismo de compo- nentes estructurales que dan firmeza a las células (Schapendonk y Brouwer, 1984) y confieren a los frutos mayor vida de almacenamiento (Ando et al., 2012; Ando y Grumet, 2010; Yong et al., 2013; Verheul et al., 2013) y capacidad de resistir impactos durante el transporte y manejo en poscosecha y mercadeo (Bourne, 1980; Muy et al., 2004; Gómez et al., 2006; Ando et al., 2012).

Una de las consecuencias de la poca firmeza de los frutos de pepino, como ocurrió con la solución al 25\% (tabla 3), es la rápida pérdida de calidad visual y sensorial, manifestada en primera instancia en marchitamiento (Suslov y Cantwell, 2012; Moreno et al., 2013), así como alta susceptibilidad a pudriciones, amarillamiento y deshidratación, las cuales se caracterizan por el desarrollo de tejido esponjoso y menor turgencia, debido a la mayor pérdida de agua de las células por transpiración, producto de la plasmólisis y la menor acumulación de azúcares en las paredes celulares (Verheul et al., 2013). En este sentido, la mayor aplicación de nutrimentos minerales favoreció características de calidad en las que son exigentes las hortalizas, ya que en términos generales dichas plantas demandan intensivamente la mayoría de nutrimentos (Muy et al., 2004; Gómez et al., 2006; Ando y Grumet, 2010; Kazemi, 2013). 
Tabla 3. Comparación de medias de firmeza de frutos de pepino en cuatro concentraciones de la solución nutritiva universal de Steiner.

\begin{tabular}{|c|c|c|c|c|c|}
\hline \multicolumn{2}{|c|}{ T1 } & T2 & T3 & T4 \\
\hline 36 & - & $45,13 \mathrm{c}$ & $49,93 \mathrm{~b}$ & $57,03 \mathrm{a}$ & 2,90 \\
\hline 42 & $42,67 \mathrm{c}$ & $55,23 \mathrm{~b}$ & $54,03 \mathrm{~b}$ & $58,70 \mathrm{a}$ & 2,11 \\
\hline 49 & $55,00 \mathrm{~b}$ & $55,57 \mathrm{~b}$ & $53,47 \mathrm{~b}$ & $65,70 \mathrm{a}$ & 4,32 \\
\hline
\end{tabular}

T (tratamientos): T1: 25\%, T2: 75\%; T3: 125\%; T4: 175\% de la solución nutritiva de Steiner; ddt: días después del trasplante; DMS: diferencia mínima significativa; CV: coeficiente de variación. Promedios con letras distintas, dentro de una misma columna, indican diferencia significativa según la prueba de Tukey $(P \leq 0,05)$.

\section{Grados Brix y acidez total titulable}

No se presentaron diferencias estadísticas entre tratamientos para estas variables (tablas 4 y 5 ).

En cuanto a grados Brix, para pepino como fruto no climatérico, no se han reportado altos valores una vez cosechados (Muy et al., 2004; Moreno et al., 2013). Los valores obtenidos en los diferentes tratamientos se encuentran dentro de los rangos indicados por Kleinhenz y Bumgarner (2012) y Anjanappa et al. (2012), y de acuerdo con Moreno et al (2013) no presentan cambios significativos durante el desarrollo del fruto, como ocurre con los frutos climatéricos. El pequeño incremento en grados Brix observado a través del tiempo (tabla 4) puede explicarse en términos de que en las etapas iniciales del crecimiento y desarrollo del fruto, la concentración total de azúcares reductores y no reductores es muy baja (Muy et al., 2004; Verheul et al., 2013), y aumenta a medida que transcurre el tiempo, y por su condición fisiológica no climatérica, posiblemente está asociado a la pérdida de humedad de los tejidos (Lodh y Pantastico, 1984).

Respecto a acidez titulable, presentó en términos generales disminución a través del tiempo para todos los tratamientos (tabla 5), lo que está de acuerdo con lo indicado por Schapendonk y Challa (1980), Gómez et al. (2006) y Kazemi (2013), en vista de que al transcurrir el desarrollo de los frutos, el proceso de madurez avanza, con una disminución del pH en el endocarpio, relacionado con pérdida de clorofila en el exocarpo e incremento del contenido de ácidos, glucosa y fructosa por encima de los de sacarosa (Handley et al., 1983; Ando y Grumet, 2013; Yong et al., 2013; Verheul et al., 2013).

Tabla 4. Comparación de medias de grados Brix de frutos de pepino en cuatro concentraciones de la solución nutritiva universal de Steiner.

\begin{tabular}{|c|c|c|c|c|c|c|}
\hline \multicolumn{2}{|c|}{ T1 } & T2 & T3 & T4 \\
\hline 36 & - & $3,43 \mathrm{a}$ & $3,37 \mathrm{a}$ & $3,77 \mathrm{a}$ & 11,01 \\
\hline 42 & $3,47 \mathrm{a}$ & $3,40 \mathrm{a}$ & $3,47 \mathrm{a}$ & $3,80 \mathrm{a}$ & 5,74 \\
\hline 49 & $3,80 \mathrm{a}$ & $3,93 \mathrm{a}$ & $3,60 \mathrm{a}$ & $4,07 \mathrm{a}$ & 4,72 \\
\hline
\end{tabular}

T (tratamientos): T1: 25\%, T2: 75\%; T3: 125\%; T4: 175\% de la solución nutritiva de Steiner; ddt: días después del trasplante; DMS: diferencia mínima significativa; CV: coeficiente de variación. Promedios con letras distintas, dentro de una misma columna, indican diferencia significativa según la prueba de Tukey $(P \leq 0,05)$. 


\section{Tabla 5. Comparación de medias de acidez total titulable de frutos de pepino en cuatro concentraciones} de la solución nutritiva universal de Steiner.

\begin{tabular}{|r|r|r|r|r|r|r|}
\hline \multicolumn{2}{|c|}{} & T1 & T2 & T3 & T4 \\
\hline 36 & - & $2,61 \mathrm{a}$ & $2,77 \mathrm{a}$ & $2,59 \mathrm{a}$ & 18,09 \\
\hline 42 & $2,35 \mathrm{c}$ & $2,46 \mathrm{bc}$ & $3,13 \mathrm{ab}$ & $3,31 \mathrm{a}$ & 9,25 \\
\hline 49 & $1,68 \mathrm{~b}$ & $2,75 \mathrm{a}$ & $3,02 \mathrm{a}$ & $3,05 \mathrm{a}$ & 11,93 \\
\hline
\end{tabular}

T (tratamientos): T1: 25\%, T2: 75\%; T3: 125\%; T4: 175\% de la solución nutritiva de Steiner; ddt: días después del trasplante; DMS: diferencia mínima significativa; CV: coeficiente de variación. Promedios con letras distintas, dentro de una misma columna, indican diferencia significativa según la prueba de Tukey $(P \leq 0,05)$.

\section{Contenido de clorofila total}

Hubo diferencias estadísticas significativas en contenido de clorofila total, con mayores valores para la solución al 175\% (tabla 6). Lo anterior se manifestó en mejor calidad de frutos, teniendo en cuenta que dicha sustancia es responsable del color verde, el cual es el índice de calidad más importante para pepinos junto con la forma y la firmeza (Booij et al., 1996; Schouten et al., 2004; Suslov y Cantwell, 2012; Yong et al., 2013), y se ha encontrado alta correlación entre el intenso color verde y la mayor cantidad de nutrimentos aplicada al cultivo (Gómez et al., 2006).

Tabla 6. Comparación de medias del contenido de clorofila total (CT), luminosidad ( $\mathrm{L}^{*}$ ) y color (coordenadas de cromaticidad $a^{*} \mathrm{y}^{*}$ ) de frutos de pepino, en cuatro concentraciones de la solución nutritiva universal de Steiner.

\begin{tabular}{|c|c|c|c|c|c|}
\hline ddt & $\mathrm{T1}$ & $\mathrm{T} 2$ & T3 & T4 & CV \\
\hline \multicolumn{6}{|c|}{ CT $\left(\mathrm{mg} \mathrm{L}^{-1}\right)$} \\
\hline 36 & - & $16,32 \mathrm{c}$ & $26,05 \mathrm{~b}$ & 34,33 a & 6,57 \\
\hline 42 & $5,57 \mathrm{c}$ & $20,50 \mathrm{~b}$ & 29,69 a & 34,48 a & 7,95 \\
\hline 49 & $12,95 \mathrm{~d}$ & $24,22 \mathrm{c}$ & $33,19 b$ & 37,96 a & 5,08 \\
\hline \multicolumn{6}{|c|}{$L^{*}$} \\
\hline 36 & - & $26,45 \mathrm{c}$ & 27,18 b & 28,63 a & 1,17 \\
\hline 42 & $25,57 \mathrm{c}$ & $26,89 \mathrm{bc}$ & $28,11 \mathrm{ab}$ & 28,49 a & 1,90 \\
\hline 49 & $25,44 d$ & $26,62 \mathrm{c}$ & $28,28 \mathrm{~b}$ & 28,78 a & 0,61 \\
\hline \multicolumn{6}{|c|}{ Coordenada de cromaticidad a* } \\
\hline 36 & - & $-4,85 b$ & $-8,72 c$ & $-13,80 d$ & 8,87 \\
\hline 42 & $-3,02$ a & $-5,63$ a & $-8,35 b$ & $-12,87 c$ & 12,49 \\
\hline 49 & $-2,21 \quad a$ & $-6,23 b$ & $-8,13 \mathrm{c}$ & $-9,79 d$ & 4,89 \\
\hline \multicolumn{6}{|c|}{ Coordenada de cromaticidad b* } \\
\hline 36 & - & 10,26 a & $7,92 \mathrm{~b}$ & $4,84 \mathrm{c}$ & 11,73 \\
\hline 42 & 7,52 а & $8,52 \mathrm{a}$ & 8,32 a & $5,59 \mathrm{~b}$ & 8,94 \\
\hline 49 & - & - & - & - & - \\
\hline
\end{tabular}

TT (tratamientos): T1: 25\%, T2: 75\%; T3: 125\%; T4: 175\% de la solución nutritiva de Steiner; ddt: días después del trasplante; DMS: diferencia mínima significativa; CV: coeficiente de variación. Promedios con letras distintas, dentro de una misma columna, indican diferencia significativa según la prueba de Tukey $(P \leq 0,05)$. 
El alto contenido de clorofila en frutos de pepino encontrado para el tratamiento con 175\% de concentración es importante además debido a que las principales pérdidas en poscosecha se relacionan con su degradación y la síntesis de xantofilas (Muy et al., 2004) y, en consecuencia, la clorofila en el fruto comienza a disminuir una vez los frutos son cosechados (Kaynas y Özelkök, 1999, Schouten et al., 2004).

\section{Luminosidad}

Presentó valores promedios más altos para el tratamiento con solución al $175 \%$, con diferencias estadísticas significativas respecto a las demás concentraciones (tabla 6). Lo anterior indicó presencia de más brillo exterior, por tanto, mejor calidad respecto a las demás concentraciones (Esmahan et al., 2005), ya que se relaciona con mayor cercanía al color blanco en el círculo cromático (McGuire, 1992; Kónica Minolta, 2003).

\section{Color (coordenadas de cromaticidad $a^{*}$ y $b^{*}$ )}

Se encontró diferencias estadísticas significativas entre tratamientos, a favor de la concentración al $175 \%$ con respecto a las demás (tabla 6 ). El comportamiento más negativo de la coordenada de cromaticidad a* para los frutos del tratamiento 4
(175\% de concentración) indica que se presentó color verde más intenso respecto a los frutos de las demás concentraciones, alejándose bastante del valor cero que corresponde a gris en el círculo cromático (Kónica Minolta, 2003).

En relación a la coordenada de cromaticidad b*, presentó menores valores para los frutos del tratamiento 4 (175\% de concentración), lo que significa que el color verde de dichos órganos estuvo bastante alejado de la dirección que en el círculo cromático conduce hacia el color amarillo, dándole mayor intensidad al verde (Ando et al., 2012; Kazemi, 2013; Yong et al., 2013; Verheul et al., 2013). En general, a los $49 \mathrm{ddt}$ se presentaron valores demasiado pequeños que indican ausencia del color amarillo en frutos (tabla 6).

\section{CONCLUSIONES}

Con el 175\% de concentración de la solución nutritiva universal de Steiner hubo mejor calidad de frutos de pepino respecto a las variables longitud y diámetro ecuatorial, firmeza, contenido total de clorofila, luminosidad y color. No hubo respuesta del cultivo de pepino a la aplicación de diferentes cantidades de nutrimentos minerales con la solución nutritiva universal de Steiner en cuanto a grados Brix y acidez total titulable de los frutos.

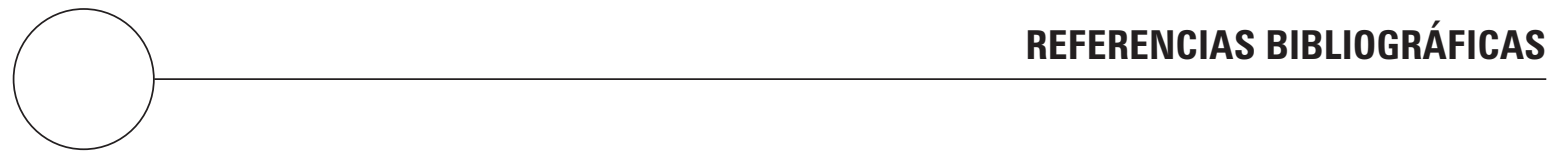

Abu, S., M. Suwwan y E. Al. 2013. The influence of plant growth regulators on callus induction from hypocotyls of cucumber (Cucumis sativus L.). Adv. Environ. Biol. 7(2), 339-343.

Ando, K., K. Carr y R. Grumet. 2012. Transcriptome analysis of early cucumber fruit growth identifies distinct gene modules associated with phases of development. BMC Genomics 13, 5-18. Doi: 10.1186/1471-2164-13-518
Ando, K. y R. Grumet. 2010. Transcriptional profiling of rapidly growing cucumber fruit by 454 -pyrosequencing analysis. J. Amer. Soc. Hort. Sci. 135(4), 291-302.

Anjanappa, M., J. Venkatesh y S. Kumara. 2012. Growth, yield an quality attributes of cucumber (Cv. Hassan local) as influenced by integrated nutrient management grown under protected condition. Veg. Sci. 39(1), 47-50. 
Banco de México. 2013. Estimación de las exportaciones agroalimentarias a nivel de entidad federativa. Secretaría de Agricultura, Ganadería, Desarrollo Rural, Pesca y Alimentación. En: http://www. sagarpa.gob.mx/agronegocios/Documents/pablo/ Documentos/Estima_Exp_Edo.pdf; consulta: abril de 2014.

Barraza A., F.V. 2012. Acumulación de materia seca del cultivo de pepino (Cucumis sativus L.) en invernadero. Temas Agrarios 17(2), 18-29.

Booij, R., P. De Willigen, A.D.H Kreuzer, A.L. Smith y A. Van Der Werf. 1996. Nitrogen balances during growth of Brussels sprouts and leeks. Acta Hort. 428, 31-41.

Bourne, M.C. 1980. Texture evaluation of horticultural crops. HortScience 15(1), 51-56.

Caicedo, L. 1993. Horticultura. Sexta edición. Facultad de Ciencias Agropecuarias. Universidad Nacional de Colombia, Palmira, Colombia. pp. 473-474.

Davies, J. y R. Kempton. 1976. Some changes in the composition of the fruit of the glasshouse cucumber (Cucumis sativus L.) during growth, maturation and senescence. J. Sci. Food Agric. 27(5), 413-418. Doi: $10.1002 /$ jsfa.2740270504

Den Nijs, A.P.M. 1980. Adaptation of the glasshouse cucumber to lower temperatures in winter by breeding. Acta Hort. 118, 65-71.

Esmahan, R., E. Hernández y G. Picado. 2005. Manual de manejo poscosecha de hortalizas. Cámara Agropecuaria y Agroindustrial de San Salvador, El Salvador. pp. 50-55.

Gómez, M.D., A. Baille, M.M. González-Real y J.M. Mercader. 2006. Dry matter partitioning of greenhouse cucumber crops as affected by fruit load. Acta Hort. 614, 573-578.

Gruda, N. 2005. Impact of environmental factors on product quality of greenhouse vegetables for fresh consumption. Crit. Rev. Plant Sci. 24(3), 227-242. Doi: $10.1080 / 07352680591008628$

Handley, L., D. Pharr y R. McFeeters. 1983. Carbohydrate changes during maturation of cucumber fruit: Implications for sugar metabolism and transport. Plant Physiol. 72, 498-502. Doi: 10.1104/ pp.72.2.498

Harborne, J.B. 1984. Phytochemical methods: a guide to modern techniques of plant analysis. $2^{\text {nd }} \mathrm{ed}$. Chapman and Hall, Londres. pp. 120-129.
Kaynas, K. y S. Özelkök. 1999. Effect of Semperfresh on postharvest behavior of cucumber (Cucumis sativus L.) and summer squash (Cucurbita pepo L.) fruits. Acta Hort. 492, 213-220.

Kazemi. M. 2013. Response of cucumber plants to foliar application of calcium chloride and paclobutrazol under greenhouse conditions. Bull. Env. Pharmacol. Life Sci. 2 (11), 15-18.

Kleinhenz, M.D. y N.R. Bumgarner. 2012. Using ${ }^{\circ}$ Brix as an indicator of vegetable quality. Linking measured values to crop management. Fact Sheet. Agriculture and Natural Resources. The Ohio State University, Columbus, $\mathrm{OH}$.

Kónica Minolta. 2003. Precise Color Communication. Color control from perception to instrumentation. Konica Minolta Photo Imaging, Ramsey, NJ.

Lodh, S. y E. Pantastico. 1984. Cambios fisicoquímicos durante el crecimiento de órganos de almacenamiento. pp. 59-76. En: Pantastico, E. (ed.). Fisiología de la postrecolección, manejo y utilización de frutas y hortalizas tropicales y subtropicales. $2^{\mathrm{a}} \mathrm{ed}$. CECSA, México DF.

Marcelis, L.F.M. 1992. Non-destructive measurements and growth analysis of the cucumber fruit. J. Hort. Sci. 67(4), 457-464.

McGuire, R. 1992. Reporting of objective color measurements. HortScience 27(12), 1254-1255.

Moreno, D., W. Cruz., E. García, A. Ibáñez, J. Barrios y B. Barrios. 2013. Cambios fisicoquímicos poscosecha en tres cultivares de pepino con y sin película plástica. Rev. Mex. Cienc. Agríc. 4(6), 909-920.

Muy, D., J. Siller, J. Díaz y B. Valdéz. 2004. Efecto de las condiciones de almacenamiento y el encerado en el estatus hídrico y la calidad poscosecha de pepino de mesa. Rev. Fitotec. Mex. 27(2), 157-165.

Qureshi, R., G. Raza y R. Asma. 2010. Ethnomedical uses of herbs from northern part of Nara desert, Pakistan. Pak. J. Bot. 42 (2), 839-851.

Schapendonk, A.H.C.M. y H. Challa. 1980. Assimilate requirements for growth and maintenance of the cucumber fruit. Acta Hort. 118, 73-82.

Schapendonk, A.H.C.M. y P. Brouwer. 1984. Fruit Growth of cucumber in relation to assimilate supply and sink activity. Sci. Hortic. 23, 21-33. Doi: 10.1016/0304-4238(84)90041-4 
Schouten, R., G. Jongbloed, L. Tijskens y O. Kooten. 2004. Batch variability and cultivar keeping quality of cucumber. Postharv. Biol. Technol. 32(3), 299-310. Doi: 10.1016/j.postharvbio.2003.12.005

Suslov, T. y M. Cantwell. 2012. Cucumber: Recommendations for maintaining postharvest quality. En: http://postharvest.ucdavis.edu/pfvegetable/CucumberPhotos/; consulta: marzo de 2014.

Tsuchida, H., N. Kozukue, G. Han, S. Choi, C. Levin y M. Friedman. 2010. Low-temperature storage of cucumbers induces changes in the organic acid content and in citrate synthase activity. Posthar- vest Biol. Technol. 58(2), 129-134. Doi: 10.1016/ j.postharvbio.2010.06.006

Verheul, M.J., R. Slimestad y L.R. Johnsen. 2013. Physicochemical changes and sensory evaluation of slicing cucumbers from different origins. Europ. J. Hort. Sci. 78(4), 176-183.

Yong, S., Y. Wang, W. Jie, X. Ling, X. Meng, H. Jun, S. Wen y G. Oin. 2013. Characterization and expression profiling of cucumber kinesin genes during early fruit development: revealing the roles of kinesis in exponential cell production and enlargement in cucumber fruit. J. Exp. Bot. 64(14), 45414557. Doi: $10.1093 / \mathrm{jxb} / \mathrm{ert} 269$ 